\title{
Plantar Pressure Distribution during Standing in Female Patients with Hip Osteoarthritis Who Underwent Total Hip Arthroplasty
}

\author{
Miura $\mathbf{M}^{1 *}$, Nagai $\mathrm{K}^{2}$, Tagomori $\mathrm{K}^{3}$, Ikutomo $\mathrm{H}^{1}$, \\ Okamura $K^{1}$, Okuno $T^{1}$, Yanamoto $A^{1}$, Nakagawa \\ $\mathbf{N}^{1}$ and Masuhara $\mathrm{K}^{1}$ \\ ${ }^{1}$ Masuhara Clinic, Tenmabashi, Kita-ku, Osaka, J apan \\ ${ }^{2}$ Department of Physical Therapy, School of \\ Rehabilitation, Hyogo University of Health Sciences, \\ Kobe, J apan \\ *Corresponding author: Namika Miura, Masuhara \\ Clinic, 3-4-2, Tenmabashi, Kita-ku, Osaka 530-0042, \\ Japan
}

Received: June 08, 2021; Accepted: July 13, 2021; Published: July 20, 2021

\begin{abstract}
Introduction: Assessment of plantar pressure indicates the manner in which the plantar region contacts the ground as the first point in a leg-linked kinetic chain, and receives force from the ground. However, few studies have examined the changes in plantar pressure distribution in patients who underwent Total Hip Arthroplasty (THA) before and after THA, or compared plantar pressure distribution between THA patients and healthy adults.
\end{abstract}

Objective: Plantar pressure distribution in patients with end-stage hip osteoarthritis who undergo THA may be adjusted to that in healthy adults by correcting leg length discrepancy. Herein, our objective was to find out if the plantar pressure distribution during standing differs before and after THA, and between healthy adults and THA patients.

Design: Case control study.

Setting: Single orthopedic clinic in Japan.

Participants: THA patients ( $n=58$; THA group) and healthy adults $(n=53$; control group).

Interventions: Not applicable.

Main outcome measure(s): The maximum plantar pressure under each foot measured during standing for $20 \mathrm{~s}$ was assessed for location, symmetry, and leg length discrepancy.

Results: The distribution plantar pressure in the THA group differed preand postoperatively. The maximum plantar pressure region was the heel in approximately $80 \%$ of the patients three months after THA; it was not different in THA patients three months postoperatively and in healthy adults. Patients with asymmetrical maximum plantar pressure regions were those whose postoperative maximum plantar pressure region in the affected leg was the forefoot and those whose maximum plantar pressure region in the affected leg shifted to the heel. The leg length discrepancies decreased significantly after THA.

Conclusions: The plantar pressure distribution during standing in female patients adjusted to that in healthy adults after THA. Patients with asymmetrical distribution of maximum plantar pressure may benefit from balance assessment and physical therapy.

Keywords: Total hip arthroplasty; Plantar pressure; Osteoarthritis

\section{Introduction}

The feet are the first and, in general, the only part of the body that contact the external environment directly during standing. In addition, the foot provides sensory information regarding contact with the ground [1,2]. A number of systems that measure foot pressure are available, but the results of one system should not generally be compared with another $[3,4]$. It may be clinically important to measure the distribution of plantar pressure rather than its value. Plantar pressure distribution can be influenced by factors such as weight, age, sex, anatomical structure of the foot, joint range of motion, and sports activities [5,6]. We have shown that the leg length discrepancy in patients with end-stage hip Osteoarthritis (OA) predicted their plantar pressure distribution. We have also shown that the plantar pressure is higher in the heel in approximately $80 \%$ of healthy women and $65 \%$ of patients with end-stage hip OA and higher in the forefoot in approximately $25 \%$ of these patients [7]. Assessment of plantar pressure indicates how the plantar region contacts the ground as the first point in a leg-linked kinetic chain and how the plantar region receives force from the ground. Periyasamy et al. described that pressure distribution measurement techniques are useful for understanding the biomechanics of the human foot and 


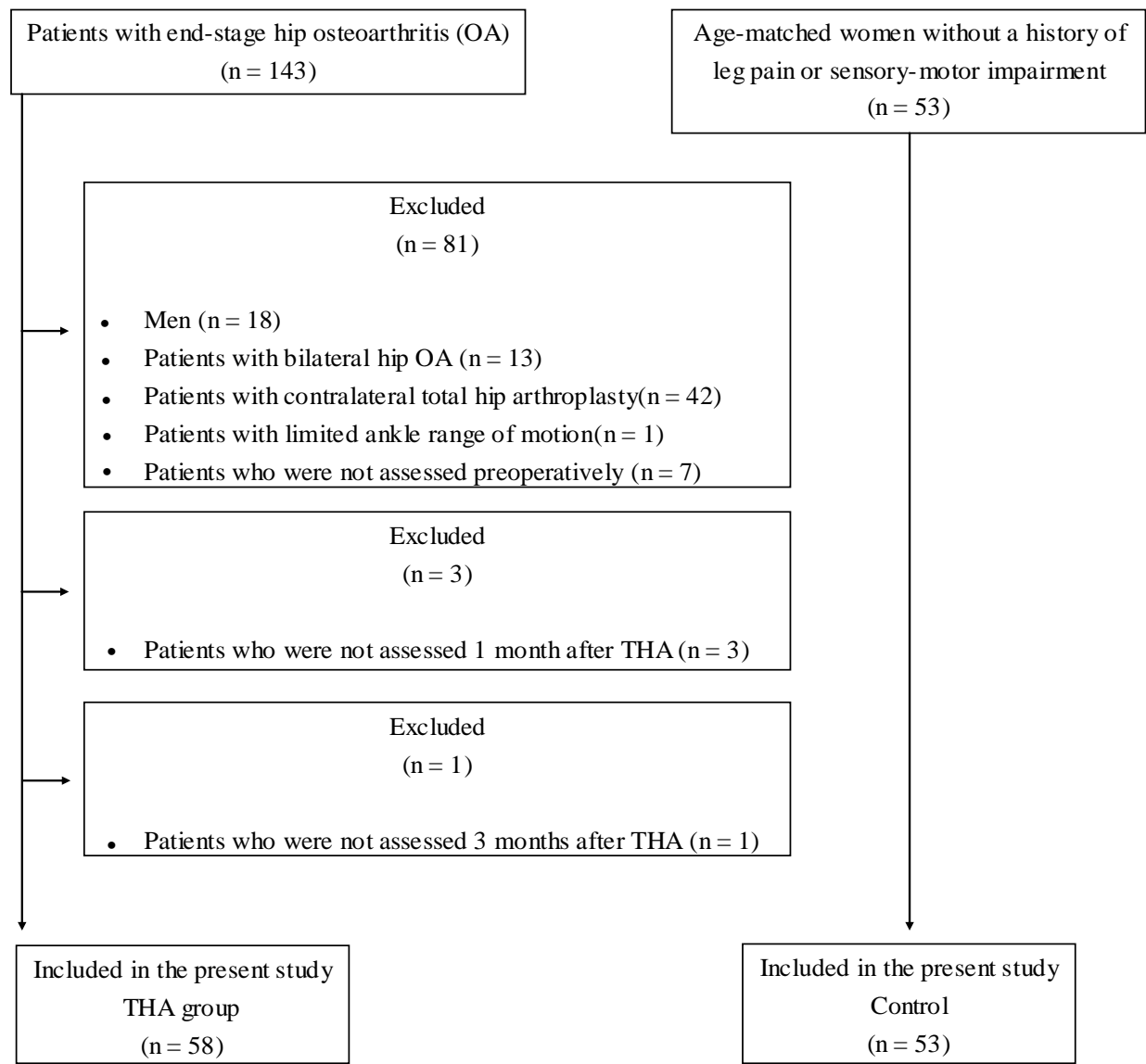

Figure 1: Flowchart of participant recruitment and enrollment.

addressing various concerns regarding associations between plantar pressure distribution and lower-extremity posture [2,5]. However, few studies have examined how plantar pressure distribution in patients who underwent Total Hip Arthroplasty (THA) changed from before THA to after THA or compared plantar pressure distribution between THA patients and healthy adults.

For this study, we hypothesized that the plantar pressure distribution during standing in patients with end-stage hip OA can be adjusted to that of healthy adults after THA resulting from correction of leg length discrepancies. Thus, this study aimed to investigate how plantar pressure distribution during standing in patients who underwent THA changed from before THA and to clarify whether plantar pressure distribution during standing in patients with THA differs from that of healthy adults.

\section{Methods}

\section{Participants}

The present study employed the case control study design. In 2016, 143 patients with end-stage hip OA (Kellgren and Lawrence Grading System: grade 4) were admitted to our institution for primary THA. Of these patients, we excluded men $(n=18)$ and selected only women to control for sex-specific differences in plantar pressure distribution [5]. In addition, we excluded the following patients: those with $\mathrm{OA}$ in both hip joints $(n=13)$, those who had undergone contralateral THA $(n=42)$, those who had severe limitation in their ankle range of motion ( $\mathrm{n}=1)$, and those who could not be assessed for any other reasons before, one month after, and three months after THA $(n=7$, $\mathrm{n}=3$, and $\mathrm{n}=1$, respectively). A total of 58 patients [mean age 62.9 \{standard deviation (SD) 9.9\} years, Body Mass Index (BMI) 22.9 (SD 3.2) $\mathrm{kg} / \mathrm{m}^{2}$; THA group] participated in this study (Figure 1).

A single surgeon performed all operations via a posterolateral approach. All patients remained in the clinic for 28 days postoperatively and received physical therapy seven days per week. Physical therapy was started on the first postoperative day and was composed of range of motion exercises, muscle strengthening, gait training, aerobic exercise, postural restoration exercise, and Activities of Daily Living (ADL) training. Patients were permitted partial weight bearing with double crutches at two days and full weight bearing with a cane at two weeks postoperatively. They were instructed to continue their tailored exercises at home. In outpatient rehabilitation, patients were assessed regarding their performance in physical function and ADLs and provided with a tailored home exercise program to address identified limitations. All patients were able to walk with a cane for $>10 \mathrm{~min}$ at four weeks postoperatively.

The control group comprised 53 age-matched women (mean age 61.2 (SD 6.8) years, BMI 21.9 (SD 3.1) $\mathrm{kg} / \mathrm{m}^{2}$ ) without a history of leg pain or sensory-motor impairment who were recruited through friends and relatives of the patients and through colleagues at our institution. 


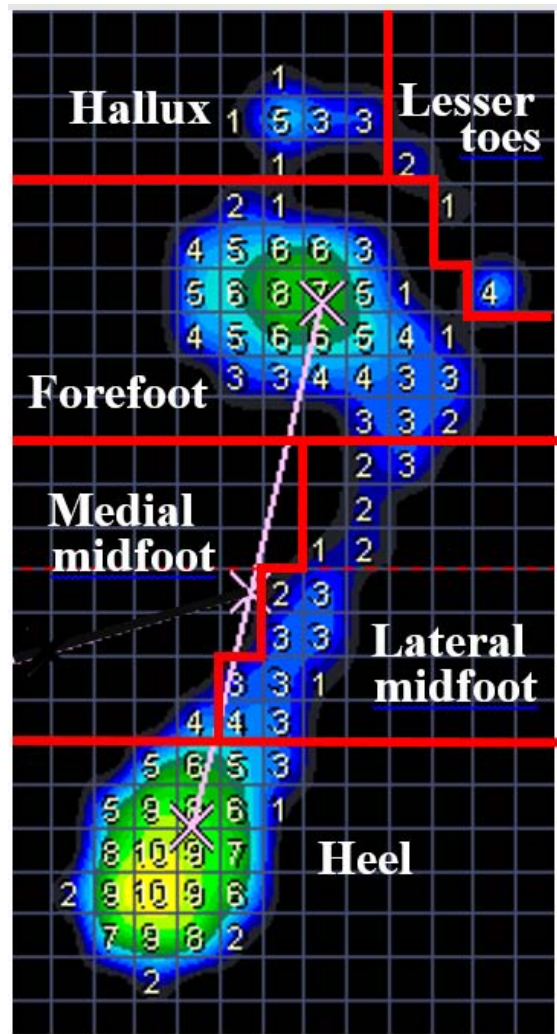

Figure 2: Graphical representation used to describe plantar pressure variables of the foot.

Plantar pressure $\left(1-14 \mathrm{~N} / \mathrm{cm}^{2}\right)$ is shown on each $1-\mathrm{cm}^{2}$ zone.

The study was approved by our institution's ethics review board (approval number 1062). We adhered to the principles outlined in the Declaration of Helsinki. All participants were informed of the procedures before they consented to participate, and all participants signed an informed consent statement.

\section{Procedures}

In all participants, the plantar pressure distribution under each foot was assessed. In the THA group, the plantar pressure was measured three times: before THA, one month after THA, and three months after THA. The Pressure Distribution Measurement Platform with PDMS software (Zebris Medical GmbH, Isnyim Allgau, Germany) was used to measure plantar pressure under each foot during standing. This platform has 1506 pressure sensors that are placed at $1 \mathrm{~cm}$ intervals on a $320 \times 470-\mathrm{mm}$ grid. Plantar pressure is shown from $1 \mathrm{~N}$ to $14 \mathrm{~N}$ on each $1 \mathrm{~cm}^{2}$ zone (Figure 2). The force platform measures vertical reaction forces of each foot. The range of measurable pressure was $1-120 \mathrm{~N} / \mathrm{cm}^{2}$, and the sampling frequency was $50 \mathrm{~Hz}$. Participants were blinded to the display of the measuring system, which showed values in percentages and the color scale of force distribution. Participants were instructed to stand for $20 \mathrm{~s}$, with their bare feet placed on the platform and their arms hanging freely at their sides. We did not attempt to control their posture any further. All participants could stand independently without assistive devices. Measurements were performed by five physical therapists with at least 10 years of clinical experience. With reference to previous studies $[1,7-$ 9], the recorded plantar region surface on the measurement platform was divided into six regions (hallux, lesser toe, forefoot, medial midfoot, lateral midfoot, and heel; Figure 2). Among the six regions, we defined the region that contained a $1 \mathrm{~cm}^{2}$ zone (this region had the highest plantar pressure) as the maximum plantar pressure region. For example, in Figure 2, the $1 \mathrm{~cm}^{2}$ zone that had the highest plantar pressure $(10 \mathrm{~N})$ was found in the heel. Accordingly, the maximum plantar pressure region was the heel. The maximum plantar pressure region was compared between the affected leg of the THA group (before THA and one and three months after THA) and the right foot of the control group. We classified the participants as having symmetrical distribution if the distribution of the maximum pressure under each foot was balanced and asymmetrical distribution if the distribution of the maximum plantar pressure was not balanced. The number of patients with symmetrical and asymmetrical distributions were compared (1) between the THA group (before THA and one and three months after THA) and the control group; (2) between those in the THA group and the control group whose maximum plantar pressure region was the heel; and (3) between those in the THA group and those in the control group whose maximum plantar pressure region was the forefoot. Furthermore, as the plantar pressure was higher in the forefoot in some patients with end-stage hip OA, we examined how the maximum plantar pressure region of the THA group, whose maximum plantar pressure region was the forefoot before THA, changed after THA. Among patients whose maximum plantar pressure region was the forefoot before THA, the proportion of patients with a symmetrical and an asymmetrical distribution was compared between patients whose maximum plantar pressure region shifted to the heel one month after THA, patients in whom the maximum plantar pressure region shifted to the heel three months after THA, and the control group, in whom maximum plantar pressure region was the heel.

In the THA group, leg length discrepancy was assessed before and one month after THA. The affected leg of patients with hip OA was shorter than the unaffected leg [10], and the leg length discrepancy in patients with end-stage hip OA predicted their plantar pressure distribution [7]. The leg length discrepancy in most patients after THA could be corrected. Leg length discrepancy was determined by measuring the difference in the perpendicular distance between a line passing through the lower edge of the teardrop points and the tip of the lesser trochanter for both legs based on anteroposterior radiographs of the pelvis in the supine position [11]. If the affected leg was shorter, leg length discrepancy was represented as a negative value. If the affected leg was longer, leg length discrepancy was represented as a positive value. The measurement was performed by a single physical therapist with at least 10 years of clinical experience.

\section{Statistics}

Data for age and BMI were tested for normality using KolmogorovSmirnov tests and evaluated for differences with independent sample t-tests. Age and BMI were compared statistically between the THA and control groups. Differences in the distribution of maximum plantar pressure between the affected leg of the THA group (before THA, one month after THA, and three months after THA) and the right foot of the control group were tested using Fisher's exact test and residual analysis. Using Fisher's exact or $\chi 2$ test and residual analysis, differences in the number of participants with symmetrical and asymmetrical distributions were compared (1) between the THA 
group (before THA and one and three months after THA) and the control group, (2) between the THA group and the control group specifically in those whose maximum plantar pressure region was the heel, (3) between the THA group and the control group specifically in those whose maximum plantar pressure region was the forefoot, and (4) between patients whose maximum plantar pressure region shifted to the heel at one month and three months after THA, and the control group participants whose maximum plantar pressure region was the heel. Differences in leg length discrepancy between before THA and one month after THA were tested using the Wilcoxon signed-rank test. All statistical analyses were performed using SPSS (IBM, Tokyo, Japan), 95\% confidence intervals (CI) were determined, and statistical significance was set at $\mathrm{p}<0.05$.

\section{Results}

The THA and control groups were not significantly different in terms of age ( $\mathrm{P}=0.297,95 \% \mathrm{CI},-4.93$ to $1.52, \mathrm{r}=0.10)$ or $\mathrm{BMI}(\mathrm{P}=0.079$, $95 \%$ CI, -2.29 to $0.13, \mathrm{r}=0.17$ ). Table 1 shows the distribution of maximum plantar pressure in the two groups. We found statistically significant differences in the distribution of maximum plantar pressure in the affected leg between the THA groups (before THA, one month after THA, and three months after THA) and the control group $(\mathrm{P}=0.029$, Cramer's $\mathrm{V}=0.15)$. One month after THA, the proportion of patients whose maximum plantar pressure region was the heel was significantly higher and the proportion of patients whose maximum plantar pressure region was the forefoot was significantly lower. Differences were not found in the proportion of patients whose maximum plantar pressure region was another region of the foot. At three months after THA, we found no significant differences in the proportion of patients whose maximum plantar pressure region was any region of the foot. The maximum plantar pressure region was the heel in approximately $80 \%$ of both the THA group and the control group.

Table 2 shows the proportion of participants with symmetrical and asymmetrical distributions in the THA group and the control group, and we found no statistically significant differences $(\mathrm{P}=0.171$, $\varphi=0.15)$. In the THA group and the control group whose maximum

Table 1: Distributions of maximum plantar pressure regions.

\begin{tabular}{|c|c|c|c|c|c|c|}
\hline THA group (Affected plantar region) & Hallux & Lesser toes & Forefoot & Lateral midfoot & Medial midfoot & Heel \\
\hline \multicolumn{7}{|l|}{ preoperative } \\
\hline $\mathrm{n}(\%)$ & $1(1.7)$ & $0(0)$ & $15(25.9)$ & $4(6.9)$ & $0(0)$ & $38(65.5)$ \\
\hline residual & -0.51 & 0 & $2.98^{*}$ & 1.33 & 0 & $-3.00^{*}$ \\
\hline \multicolumn{7}{|l|}{1 month postoperative } \\
\hline n (\%) & $2(3.4)$ & $0(0)$ & $2(3.4)$ & $1(1.7)$ & $0(0)$ & $53(91.4)$ \\
\hline residual & 0.44 & 0 & $-2.70^{*}$ & -1.01 & 0 & $2.63^{*}$ \\
\hline \multicolumn{7}{|l|}{3 months postoperative } \\
\hline n (\%) & $1(1.7)$ & $0(0)$ & $7(12.1)$ & $2(3.4)$ & $0(0)$ & $48(82.8)$ \\
\hline residual & -0.51 & 0 & -0.51 & -0.23 & 0 & 0.75 \\
\hline \multicolumn{7}{|l|}{ Control group (Right plantar region) } \\
\hline n (\%) & $2(3.8)$ & $0(0)$ & 8 (15.1) & $2(3.8)$ & $0(0)$ & $41(77.4)$ \\
\hline residual & 0.59 & 0 & 0.24 & -0.08 & 0 & -0.4 \\
\hline
\end{tabular}

Fisher's exact test indicated a significant difference, $P=0.029$

${ }^{*}$ Residual analysis indicated a significant difference, $\mathrm{P}<0.05$

THA, total hip arthroplasty
Table 2: Proportion of participants with symmetrical and asymmetrical distributions in the THA and control groups.

\begin{tabular}{|c|c|c|}
\hline \multicolumn{1}{|c|}{ THA group } & $\begin{array}{c}\text { Symmetrical } \\
\text { distribution }\end{array}$ & $\begin{array}{c}\text { Asymmetrical } \\
\text { distribution }\end{array}$ \\
\hline preoperative & $37(63.8)$ & $21(36.2)$ \\
\hline $\mathrm{n}(\%)$ & $37(63.8)$ & $21(36.2)$ \\
\hline $\mathbf{1}$ month postoperative & & \\
\hline $\mathrm{n}(\%)$ & $38(65.5)$ & $20(34.5)$ \\
\hline $\begin{array}{l}3 \text { months } \\
\text { postoperative }\end{array}$ & & $10(19.2)$ \\
\hline $\mathrm{n}(\%)$ & $42(80.8)$ & \\
\hline Control group & $\mathrm{n}(\%)$ & \\
\hline
\end{tabular}

$\mathrm{X}^{2}$ test did not indicate a significant difference, $\mathrm{P}=0.171$

THA, total hip arthroplasty

Table 3: Proportion of participants with symmetrical and asymmetrical distributions in the THA and control groups with the heel as the maximum plantar pressure region.

\begin{tabular}{|c|c|c|}
\hline \multicolumn{1}{|c|}{ THA group } & $\begin{array}{c}\text { Symmetrical } \\
\text { distribution }\end{array}$ & $\begin{array}{c}\text { Asymmetrical } \\
\text { distribution }\end{array}$ \\
\hline preoperative & $34(89.5)$ & $4(10.5)$ \\
\hline $\mathrm{n}(\%)$ & $37(69.8)$ & $16(30.1)$ \\
\hline $\mathbf{1}$ month postoperative & & \\
\hline $\mathrm{n}(\%)$ & $37(77.1)$ & $11(22.9)$ \\
\hline $\begin{array}{l}\mathbf{3} \text { months } \\
\text { postoperative }\end{array}$ & & \\
\hline $\mathrm{n}(\%)$ & $35(85.4)$ & $6(14.6)$ \\
\hline Control group & $\mathrm{n}(\%)$ & \\
\hline
\end{tabular}

Fisher's exact test did not indicate a significant difference, $P=0.084$

THA, total hip arthroplasty

plantar pressure region was the heel, the difference in the proportion of participants with symmetrical and asymmetrical distributions was not statistically significant $(\mathrm{P}=0.084, \varphi=0.19)$ (Table 3 ). Table 4 shows the proportion of participants with symmetrical and asymmetrical distributions in the THA group and the control group whose maximum plantar pressure region was the forefoot. In the control 
Table 4: Proportion of participants with symmetrical and asymmetrical distributions in the THA and control groups with the forefoot as the maximum plantar pressure region.

\begin{tabular}{|l|c|c|}
\hline \multicolumn{1}{|c|}{ THA group } & $\begin{array}{c}\text { Symmetrical } \\
\text { distribution }\end{array}$ & $\begin{array}{c}\text { Asymmetrical } \\
\text { distribution }\end{array}$ \\
\hline preoperative & $3(20.0)$ & $12(80.0)$ \\
\hline $\mathrm{n}(\%)$ & -1.29 & 1.29 \\
\hline residual & $0(0)$ & $2(100)$ \\
\hline $\mathbf{1}$ month postoperative & -0.98 & 0.98 \\
\hline $\mathrm{n}(\%)$ & $1(14.3)$ & $6(85.7)$ \\
\hline residual & -1.1 & 1.1 \\
\hline $\begin{array}{l}\text { p months } \\
\text { postoperative }\end{array}$ & & $2(25.0)$ \\
\hline $\mathrm{n}(\%)$ & $6(75.0)$ & $-3.08^{*}$ \\
\hline residual & $3.08^{*}$ & \\
\hline Control group & $\mathrm{n}(\%)$ & \\
\hline residual & & \\
\hline
\end{tabular}

Fisher's exact test indicated a significant difference, $P=0.018$

*Residual analysis indicated a significant difference, $\mathrm{P}<0.05$.

THA, total hip arthroplasty.

group, the proportion of participants with symmetrical distribution was significantly higher and the proportion of patients with asymmetrical distribution was significantly lower $(\mathrm{P}=0.018, \varphi=0.56)$.

In the THA group, 15 patients had the forefoot as the maximum plantar pressure region, 12 had their maximum plantar pressure region shifted to the heel by one month after THA, and a further 10 had a shift to the heel by three months after THA. A comparison of the patients whose maximum plantar pressure region shifted to the heel by one month after THA, those whose maximum plantar pressure region shifted to the heel by three months after THA, and the control group whose maximum plantar pressure region was the heel showed that the proportion of participants with symmetrical distributions was significantly higher and the proportion of participants with asymmetrical distributions was significantly lower in the control group whose maximum plantar pressure region was the heel $(\mathrm{P}=0.048, \varphi=0.29)$ (Table 5).

The mean leg length discrepancy before THA was $-1.1 \mathrm{~cm}$ (SD

$0.91,95 \%$ CI, -1.34 to -0.86 ) and decreased significantly by one month after THA to $0.2 \mathrm{~cm}$ (SD 0.70, 95\% CI, 0.03 to 0.40$)(\mathrm{P}<0.01, \mathrm{r}=-0.86)$.

\section{Discussion}

This study indicated that the plantar pressure distribution in the affected leg during standing in female patients with end-stage hip OA changed after THA. Moreover, results showed that the maximum plantar pressure region in approximately $80 \%$ of the patients by three months after THA was the heel, and no differences were found between the THA group three months after THA and healthy adults. The maximum plantar pressure region of most healthy adults was the heel. As the leg length discrepancy decreases, the heel is more frequently observed to be the maximum plantar pressure region [7]. In this study, the leg length discrepancy in patients with hip OA decreased after THA. We interpreted that the number of patients whose maximum plantar pressure region was the heel increased after THA because the leg length discrepancy was corrected, and no differences were found in the distribution of the maximum plantar pressure between healthy adults and patients three months after THA. The relationship between plantar pressure distribution and functional leg length discrepancy may influence the change in the distribution of maximum plantar pressure between one month after THA and three months after THA.

Leg length discrepancy can be divided into two etiological aspects: a structural leg length discrepancy, those with shortening of bony structures, and a functional leg length discrepancy, those that are a result of pelvic obliquity due to hip contracture or scoliosis [12-16]. In this study, we measured structural leg length discrepancy. Nakanowatari et al. reported that $28 \%$ of their patients had functional leg length discrepancy of $\geq 5 \mathrm{~mm}$ and structural leg length discrepancy of $<5 \mathrm{~mm}$ at three weeks postoperatively [16]. We interpreted that adjusting pelvic obliquity three months after THA might correct the functional leg length discrepancy and changes in the distribution of plantar pressure result from adaptation to the structural leg length discrepancy. Assessing and adjusting pelvic obliquity through physical therapy may be able to change plantar pressure distribution earlier. This study showed that the maximum plantar pressure region under each foot was also symmetrical in $85 \%$ of healthy adults and in $>70 \%$ of the THA patients whose maximum plantar pressure region was the heel. However, the maximum plantar

Table 5: Proportion of participants with symmetrical and asymmetrical distributions in the THA group whose maximum plantar pressure region shifted to the heel after THA, and in the control group whose maximum plantar pressure region was the heel.

\begin{tabular}{|c|c|c|}
\hline THA group & Symmetrical distribution & Asymmetrical distribution \\
\hline \multicolumn{3}{|c|}{ Patients whose maximum plantar pressure region shifted to the heel 1 month after THA } \\
\hline $\mathrm{n}(\%)$ & $7(58.3)$ & $5(41.7)$ \\
\hline residual & -1.61 & 1.61 \\
\hline \multicolumn{3}{|c|}{ Patients whose maximum plantar pressure region shifted to the heel 3 months after THA } \\
\hline $\mathrm{n}(\%)$ & $6(60.0)$ & $4(40.0)$ \\
\hline residual & -1.31 & 1.31 \\
\hline \multicolumn{3}{|c|}{ Control group whose maximum plantar pressure region was the heel } \\
\hline $\mathrm{n}(\%)$ & $35(85.4)$ & $6(14.6)$ \\
\hline residual & $2.33^{*}$ & $-2.33^{*}$ \\
\hline
\end{tabular}

Fisher's exact test indicated a significant difference, $\mathrm{P}=0.048$

${ }^{*}$ Residual analysis indicated a significant difference, $\mathrm{P}<0.05$.

THA, total hip arthroplasty. 
pressure region was symmetrical in $75 \%$ of healthy adults and in $<20 \%$ of the patients whose maximum plantar pressure region was the forefoot. This indicated that the symmetry of the distribution of maximum plantar pressure under each foot in patients whose maximum plantar pressure region in the affected leg was the forefoot was different from that in healthy adults. Furthermore, in patients whose maximum plantar pressure region in the affected leg shifted from the forefoot to the heel after THA, their distribution of maximum plantar pressure tended to be asymmetrical. The position of the maximum plantar pressure region resolves the location of the Center of Pressure (COP). The COP is related strongly to balancing ability. The ability to maintain the COP within the base of support can also estimate balancing ability well [17-20]. Identifying the COP location indicates where the forces are distributed under the foot and provides insight into the postural-control strategy being used [21]. Standing is the base of many ADLs, such as walking. Assessing standing in fine detail could contribute to the body of knowledge on abnormal standing performance in patients. Although the loading of the affected leg during standing reduced in patients with hip $\mathrm{OA}$, we found that the loading of the operated leg was restored one month after THA and was maintained three months after THA [22]. However, three months after THA, patients were able to exert load on the operated leg sufficiently, and their plantar pressure distribution was not different from that of a healthy adult. Thus, we should focus on the asymmetry of the plantar pressure distribution and the COP location in patients whose maximum plantar pressure region in the affected leg is the forefoot or those whose maximum plantar pressure region in the affected leg shifts after THA. A previous study observed major post-surgical improvements compared to preoperative status, although postural balance impairment could still be noted compared with healthy controls [23]. Therefore, we should investigate whether the characteristics of plantar pressure distribution in THA patients is related to their balancing ability.

\section{Study Limitations}

In this study, we assessed the plantar pressure until three months after THA, but these results may not reflect the long-term distribution of plantar pressure after THA. Moreover, the results cannot be generalized across the population of THA recipients, as our study examined female patients only. In addition, we did not measure functional leg length discrepancy and pelvic obliquity. We suggest an investigation of the relationship between alignment in patients after THA and their plantar pressure distribution. Furthermore, we did not measure the anatomical structure of the foot and joint range of motion, or investigate sports activities. These may have influenced the plantar pressure distribution.

\section{Conclusions}

The plantar pressure distribution in the affected leg during standing in female patients with end-stage hip OA changed following THA. As a result of corrections to their leg length discrepancies, the maximum plantar pressure region shifted to the heel in $80 \%$ of the patients by three months after THA. The plantar pressure distribution in patients three months after THA was not different from that in healthy adults. Patients whose distribution of maximum plantar pressure were asymmetrical after THA would need to have their balancing ability assessed and their plantar pressure distribution adjusted to more closely match that of a healthy adult through physical therapy.

\section{Author Contributions}

Namika Miura: Conceptualization, Data curation, Methodology, Formal analysis, Investigation, Writing - original draft. Kotatsu Nagai: Conceptualization, Formal analysis, Investigation, Writingreview \& editing. Keiichi Tagomori: Conceptualization, Data curation, Investigation, Writing-review \& editing. Hisashi Ikutomo: Conceptualization, Data curation, Investigation, Writing-review \& editing. Kenichi Okamura: Conceptualization, Data curation. Takato Okuno: Conceptualization, Data curation. Asuka Yanamoto: Conceptualization. Norikazu Nakagawa: Conceptualization, Writingreview \& editing. Kensaku Masuhara: Conceptualization, Writing review \& editing, Supervision.

\section{Acknowledgements}

We would like to thank Editage (www.editage.jp) for English language editing.

\section{Funding}

This research did not receive any specific grant from funding agencies in the public, commercial, or not-for-profit sectors.

\section{References}

1. MA Nurse, BM Nigg. The effect of changes in foot sensation on plantar pressure and muscle activity, Clin. Biomech. 2001; 16: 719-727.

2. R. Periyasamy, S. Anand. The effect of foot arch on plantar pressure distribution during standing, J. Med. Eng. Technol. 2013; 37: 342-47.

3. J Hughes, S Kriss L. Klenerman, A clinician's view of foot pressure: a comparison of three different methods of measurement, Foot Ankle. 1987; 7: $277-284$

4. J Hughes, P Clark, K Linge, L Klenerman. A comparison of two studies of the pressure distribution under the feet of normal subjects using different equipment, Foot Ankle. 1993; 14: 514-519.

5. R Periyasamy, A Mishra, S Anand, AC Ammini. Preliminary investigation of foot pressure distribution variation in men and women adults while standing. Foot (Edinb). 2011; 21: 142-148.

6. D Pomarino, A Pomarino. Plantar static pressure distribution in healthy individuals: percentiles for the evaluation of forefoot loading, Foot Ankle Spec. 2014; 7: 293-297.

7. N Miura, K Nagai, K Tagomori, H Ikutomo, K Okamura, T Okuno, et al. Plantar pressure distribution during standing in women with end-stage hip osteoarthritis, Gait Posture. 2020; 76: 39-43.

8. ML Voronov, MS Pinzur, HH Hoffman, RM Havey, G Carandang, AG Patwardhan. Static measure of foot loading, Foot Ankle Spec. 2009; 2: 267 270.

9. S Saito, K. Okada, T Nishi, M Wakasa, A Saito, K Sugawara, et al. Foot pressure pattern and its correlation with knee range of motion limitations for individuals with medial knee osteoarthritis, Arch. Phys. Med. Rehabil. 2013; 94: 2502-2508.

10. H Fujimaki, Y Inaba, N Kobayashi, T Tezuka, Y Hirata, T Saito. Leg length discrepancy and lower limb alignment after total hip arthroplasty in unilateral hip osteoarthritis patients. J Orthop Sci. 2013; 18: 969-976.

11. M Kjellberg, B Al-Amiry, E Englund, GO Sjödén, AS Sayed-Noor. Measurement of leg length discrepancy after total hip arthroplasty. The reliability of a plain radiographic method compared to CT-scanogram, Skeletal Radiol. 2012; 41: 187-191.

12. R.L. Blake, H. Ferguson, Limb length discrepancies. J Am Podiatr Med 
Assoc. 1992; 82: 33-38.

13. WD Abraham, JH Dimon $3^{\text {rd }}$, Leg length discrepancy in total hip arthroplasty, Orthop. Clin. North Am. 23 (1992) 201-209.

14. B. Gurney, Leg length discrepancy, Gait Posture. 2002; 15: 195-206.

15. J Ireland, L Kessel. Hip adduction/abduction deformity and apparent leglength inequality. Clin Orthop Relat Res. 1980: 156-157.

16. T Nakanowatari, Y Suzukamo, T Suga, A Okii, G Fujii, SI Izumi. True or apparent leg length discrepancy: which is a better predictor of short-term functional outcomes after total hip arthroplasty? J Geriatr Phys Ther. 2013 36: 169-174

17. J Howcroft, ED Lemaire, J Kofman, C Kendell. Understanding responses to gait instability from plantar pressure measurement and the relationship to balance and mobility in lower-limb amputees. Clin Biomech. 2015; 320: 241 248 .

18. SL Pavão, GS Nunes, AN Santos, NACF Rocha. Relationship between static postural control and the level of functional abilities in children with cerebral palsy. Braz J Phys Ther. 2014; 18: 300-307.
19. M Nakamura, A Urashima, T Toriyama, T Ninomiya, N Fukumoto, Y Aiboshi. The visualization system of center of pressure and base of support in sitto-stand movement; Proceedings of the IEEE 4th Global Conference on Consumer Electronics; Osaka, Japan. 2015; 502-503.

20. CW Huang, PD Sue, MF Abbod, BC Jiang, JS Shieh. Measuring center of pressure signals to quantify human balance using multivariate multiscale entropy by designing a force platform, Sensors (Basel). 2013; 13: 1015110166

21. M Mettler, L Chinn, SA Saliba, PO McKeon, J Hertel. Balance training and center-of-pressure location in participants with chronic ankle instability. J Athl Train. 2015; 50: 343-349.

22. N Miura, K Tagomori, H Ikutomo, N Nakagawa, K Masuhara. Leg loading during quiet standing and sit-to-stand movement for one year after total hip arthroplasty, Physiother. Theory Pract. 2018; 34: 529-533.

23. F. de Lima, D.A. Fernandes, G. Melo, de M Roesler CR, de S Neves F, F.R. Neto, Effects of total hip arthroplasty for primary hip osteoarthritis on postural balance: a systematic review, Gait Posture. 2019; 73: 52-64 